\title{
Irreducible Posterolateral Fracture Dislocation of the Knee in an Elderly Patient Treated with Rotating Hinge Total Knee Replacement
}

\author{
Gabriele Bernardi $^{1}$ Maria Teresa Manisera ${ }^{1} \quad$ Alexander Vallone $^{1}$ Cosimo Tudisco $^{10}$ \\ ${ }^{1}$ Department of Orthopedic Surgery, University of Rome Tor Vergata, \\ Rome, Italy \\ Address for correspondence Gabriele Bernardi, MD, Department of \\ Orthopaedic Surgery, University of Rome Tor Vergata, Viale Oxford \\ 81, 00133 Rome, Lazio, Italy (e-mail: bernardi.gab@gmail.com).
} Joints 2019;7:209-214.

\begin{abstract}
Knee dislocation is one of the few real orthopaedic emergencies. It is a serious but relative uncommon injury, representing less than $0.02 \%$ of all orthopaedic injuries. Especially posterolateral knee dislocation is much rarer condition that is often missed

Keywords or misdiagnosed. The main feature of this very rare condition is to be irreducible by

- knee dislocation

- posterolateral knee dislocation

- irreducible fracture dislocation

- rotating hinge knee replacement closed reduction due to the interposition of soft tissues inside the enlarged medial joint space, such as the medial capsule and retinaculum, vastusmedialis, and medial meniscus. The pathognomonic sign of a posterolateral knee dislocation is the anteromedial distal thigh transverse "pucker" or "dimple sign." We report the case of an 87year-old man who sustained an irreducible posterolateral fracture dislocation of the knee after being hit by car which has been treated with rotating hinge knee replacement with 1-year of follow-up.
\end{abstract}

\section{Introduction}

Knee dislocation is an uncommon injury in orthopaedics, with a high rate of associated vascular and neurologic injuries, as well as potentially limb-threatening complications. The incidence is low and it has been reported less of $0.02 \%$ of musculoskeletal trauma, ${ }^{1,2}$ even if the real incidence is underestimated by spontaneous reductions and missed diagnosis. Posterolateral knee dislocation is a much rare condition, generally caused by high-energy trauma in which the medial femoral condyle buttonholes through the medial capsule and retinaculum and the tibia dislocate posterolateral, determining the "dimple" or "pucker" sign. This kind of rare knee dislocation is not reducible by closed manipulation due to the interposition of soft tissues, including the medial capsule and retinaculum, ${ }^{3}$ vastus medialis, ${ }^{4,5}$ adductor magnus, ${ }^{6}$ and medial meniscus, ${ }^{7}$ inside the enlarged medial joint space. The majority of knee dislocation is not associated with bony avulsion or fracture. Only three reports have been published in the literature describing irreducibility of a fracture dislocation of the knee with closed techniques. In the first case, a medial femoral condylar fragment was locked behind the medial tibial plateau preventing reduction. ${ }^{8}$ In second case, the patella tendon was incarcerated within the Hoffa fracture. ${ }^{9}$ In the third case, the reduction of the knee was not possible due to the incarceration of the medial meniscus within medial tibial plateau fracture. ${ }^{10}$

\section{Case Presentation}

An 87-year-old man was admitted to our emergency department of our hospital after being hit by car on his right knee while crossing the road. The primary examination revealed no life-threatening conditions. The clinical examination received

April 8, 2018

accepted

April 18, 2021

published online

June 18, 2021
DOI https://doi.org/ 10.1055/s-0041-1730980. ISSN 2282-4324.

\footnotetext{
(C) 2021. The Author(s).

This is an open access article published by Thieme under the terms of the Creative Commons Attribution-NonDerivative-NonCommercial-License, permitting copying and reproduction so long as the original work is given appropriate credit. Contents may not be used for commercial purposes, or adapted, remixed, transformed or built upon. (https://creativecommons.org/ licenses/by-nc-nd/4.0/) Georg Thieme Verlag KG, Rüdigerstraße 14, 70469 Stuttgart, Germany
} 


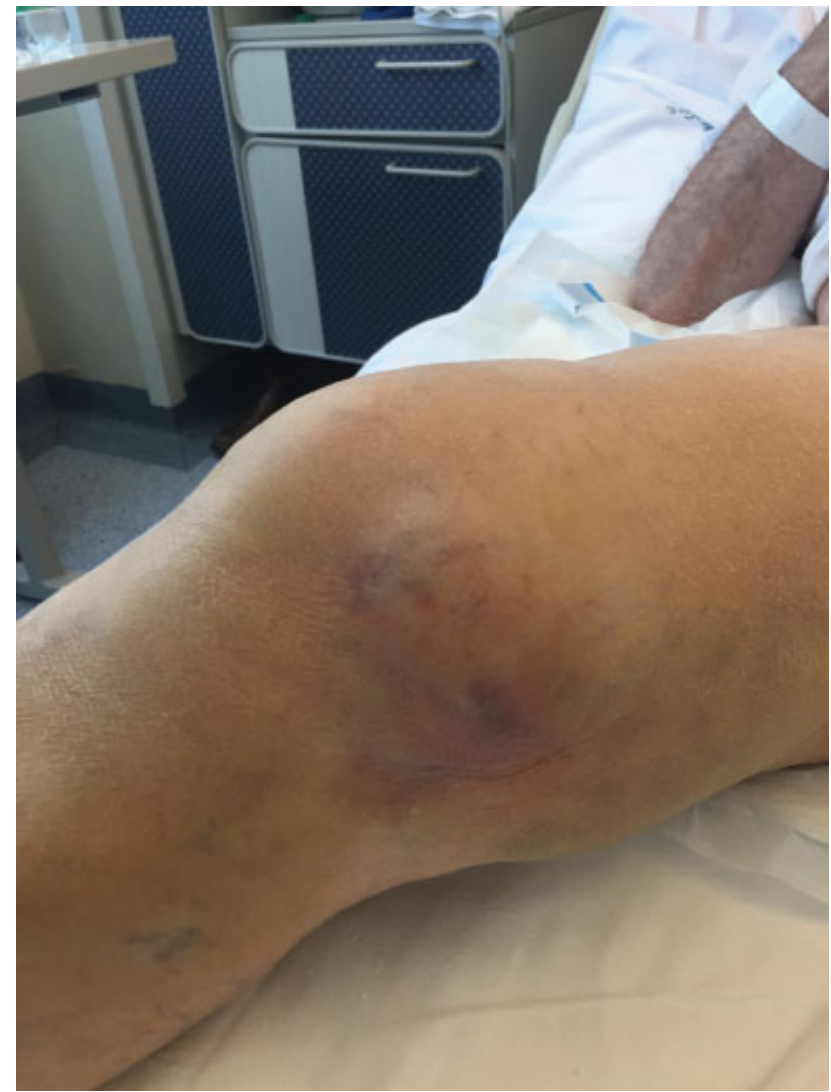

Fig. 1 Clinical picture of the medial side of the knee: the medial femoral condyle was just above the "pucker."

revealed the right knee held in a slight flexion by 30 degrees and valgus deviation of 15 degrees. There was a diffuse tenderness around the knee with a transverse ecchymotic "puckering" of the skin over the anteromedial thigh; the medial femoral condyle was palpable just above the "pucker" (-Fig. 1). No peripheral sensory and motor nerve deficits were present and dorsal pedis and posterior tibial artery pulses were normal. It was impossible to evaluate the ligamentous stability of the knee.
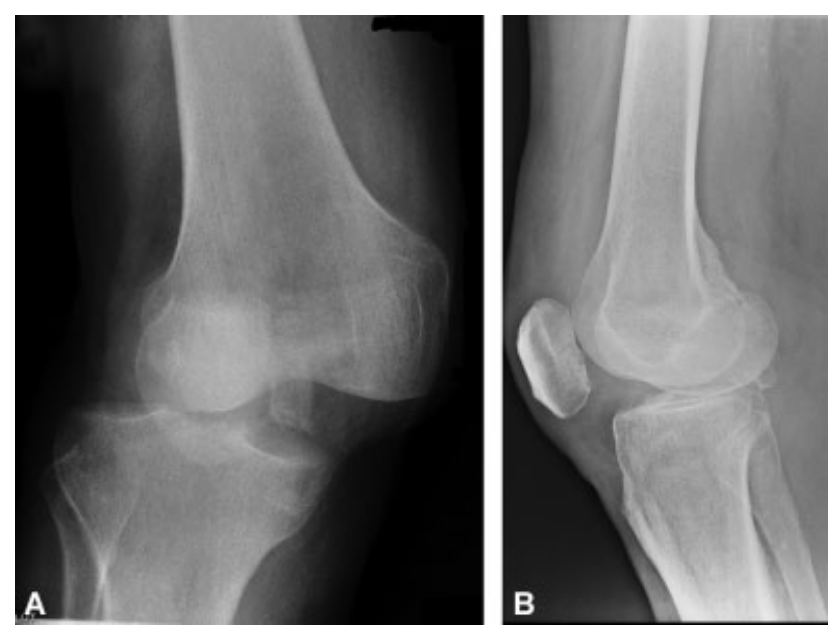

Fig. 2 (A, B) Plain anteroposterior and lateral radiographs of the right knee. AP, anteroposterior.

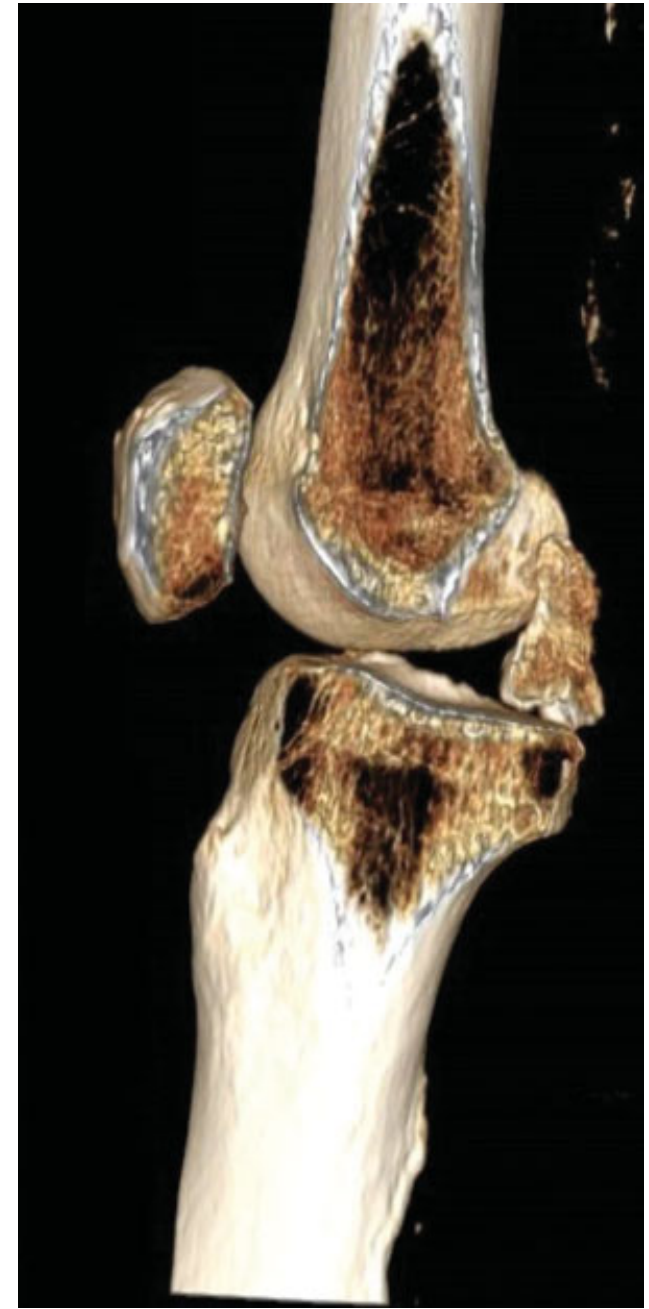

Fig. 3 CT scan with 3D reconstruction of the right knee. 3D, threedimensional; $\mathrm{CT}$, computed tomography.

Plain anteroposterior and lateral radiographs ( - Fig. 2A,B) and computed tomography (CT) with three-dimensional (3D) reconstructions ( - Fig. 3 ) demonstrated a wider medial joint space, tibial posterolateral displacement with valgus deviation, a posterior cruciate avulsion fracture of the tibia, and an osteochondral fracture of the lateral femoral condyle of the right knee (KD-V according to Schenck's classification ${ }^{11}$ ). Magnetic resonance imaging (MRI) demonstrates complete tear of the anterior cruciate ligament $(\mathrm{ACL})$, medial collateral ligament (MCL), posterolateral corner (PLC), and PCL avulsion with bony fragment. Arterial Doppler examination and CT angiography of the lower limb were found to be negative (-Fig. 4).

On the same day, a closed reduction was attempted under general anesthesia. The knee was flexed and valgus stress and internal rotation of the lower thigh were applied according to Tateda et $\mathrm{al}^{12}$ but the attempt was unsuccessful. During general anesthesia, it was possible to stress the knee up to 45 degrees of valgus deviation (-Fig. 5). Subsequently, the knee was immobilized in a plastic cast and surgery was planned for the next day.

Considering the clinical-radiographic injury patter, and especially the old age of our patient, we decided to perform a 


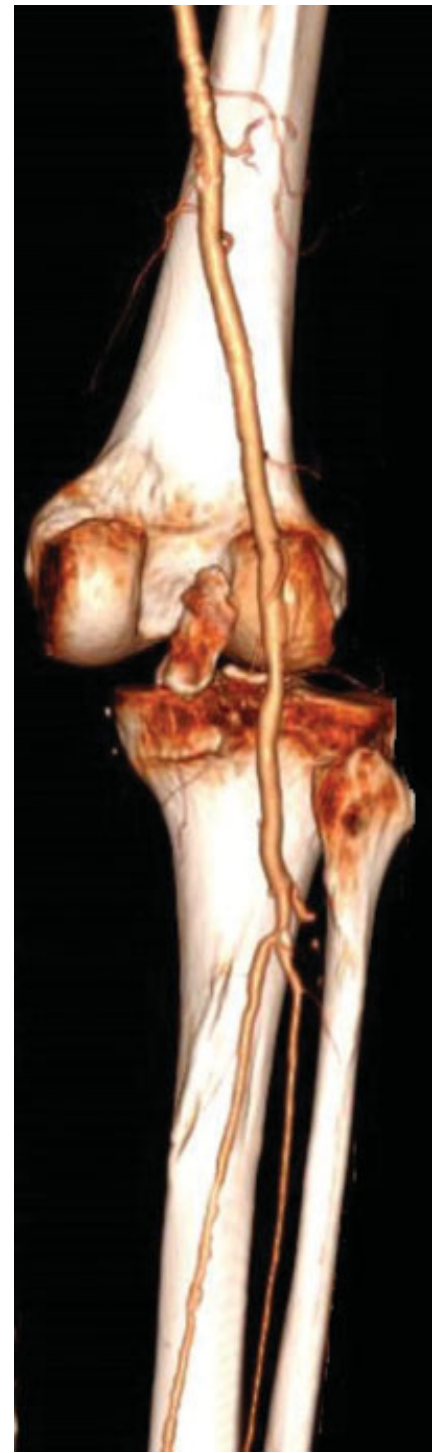

Fig. 4 CT angiography of the lower limb showed no lesion to the popliteal artery. CT, computed tomography.

rotating hinge knee replacement, instead an open reduction and multiligament reconstruction. Under spinal anesthesia and with a pneumatic tourniquet inflated on the right thigh, a standard midline incision with a medial parapatellar approach was utilized. A complete midsubstance tear of the ACL, posterior cruciate avulsion fracture with the bony fragment dislocated and rotated by 90 degrees in the intercondylar notch were found. The medial retinaculum and capsule were torn along with the distal end of the vastusmedialis and the MCL was completely avulsed from the femoral footprint. Rotating hinge knee implant (NexGen RH Knee, Zimmer; Warsaw, Indiana, United States) with stemmed femoral and tibial component was used (-Fig. 6). After the definitive component implant, the MCL was reinserted to the femoral footprint using a $2.9-\mathrm{mm}$ suture anchor (Juggerknot, Biomet; Warsaw, Indiana, United States); the medial retinaculum and the capsule were also repaired with absorbable suture.

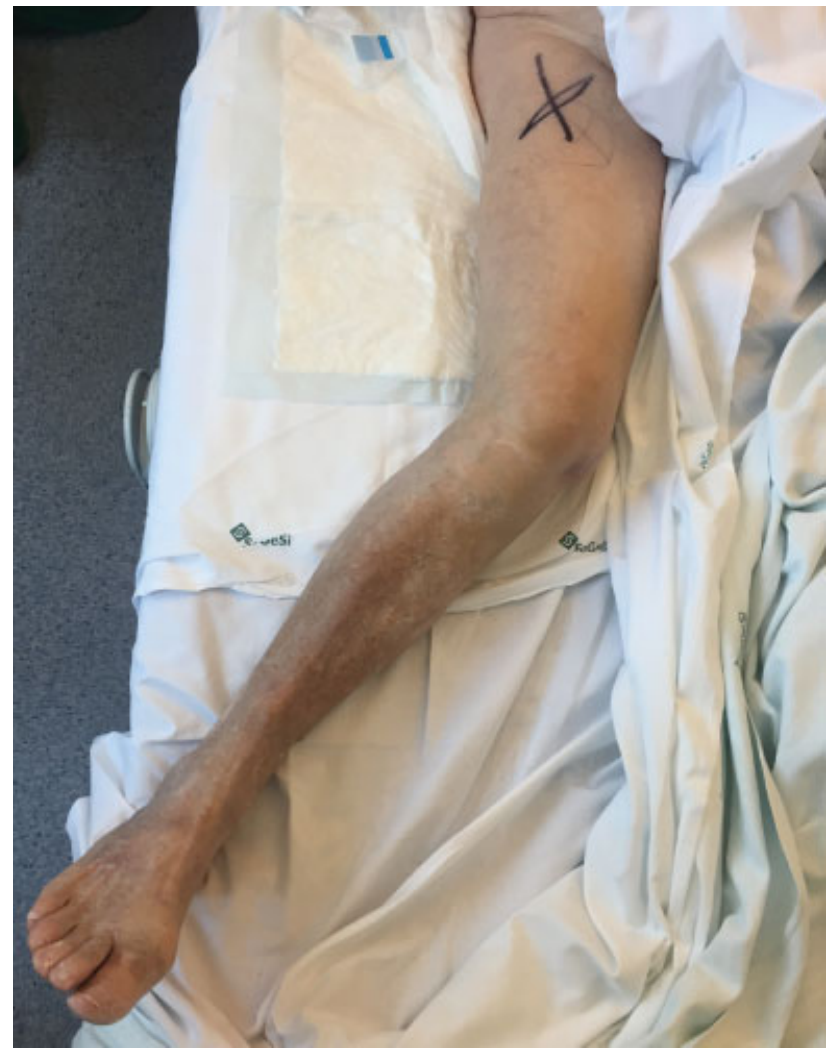

Fig. 5 Clinical picture of the angular valgus deviation of the right knee.

Rehabilitation during hospital stay was the same of standard elective total knee arthroplasty (TKA) scheme and included continuous passive motion (CPM) and early walking rehabilitation without brace. The patient received antithrombotic prevention with low molecular weight heparin (LMWH) and stockings for 4 weeks after surgery. The patient was discharged from the hospital after 7 days.

Regular follow-up visits were scheduled. At the 12months of follow-up, the patient had 0-120 degrees of painless range of motion (ROM), very good stability and no sign of osteolysis on plain radiographs (-Fig. 7A,B). The patient was very satisfied. The Oxford knee score (OKS) scored 40, the Knee Society Score (KSS) knee was 79, KSS functional was 80 .

\section{Discussion}

Posterolateral knee dislocation is a very rare subgroup of knee dislocations in which the medial femoral condyle buttonholes through the medial capsule and retinaculum and the tibia dislocates posterolateral. It represents nearly $4 \%$ of all knee dislocations. ${ }^{13}$ The main feature of this kind of injury is to be irreducible by closed reduction due to the interposition of soft tissues inside the enlarged medial joint space. The most effective mechanism for irreducible posterolateral knee dislocation is usually considered a valgus force applied to a flexed knee with tibial external 

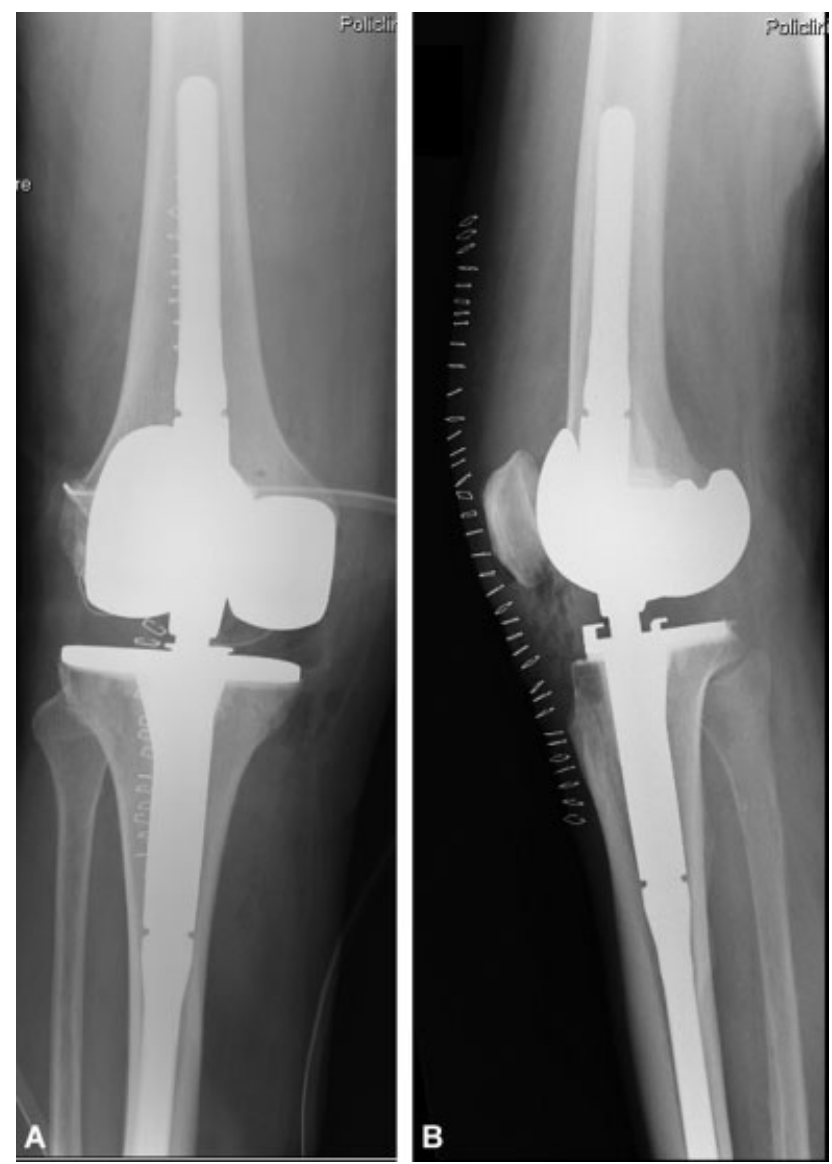

Fig. $6(A, B)$ Postoperative $X$-rays with rotating hinge prosthesis implanted.

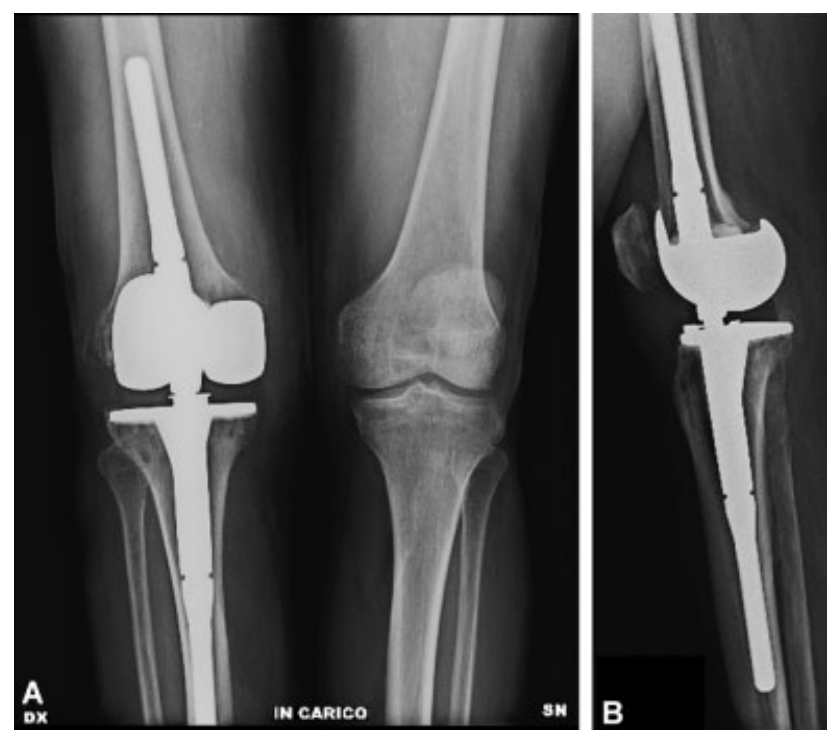

Fig. 7 (A, B) 12 months post-operative weight bearing X-rays.

rotation, ${ }^{3,14}$ and it can be sustained from high- or low-energy trauma, especially in obese patients. ${ }^{15,16}$

Knee dislocation may cause several associated lesions and potentially limb-threatening complications, including multiligamentous knee injury (disruption of more than three of the four major ligaments of the knee ${ }^{17}$ ), vascular or neurological injury, and periarticular fractures.

Knee dislocations are classified according to the anatomical classification system by Schenck and subsequently modified by Wascher. In this system, knee dislocations are divided into five groups based on which ligaments are injured, presence of fracture and associated vascular or neurologic injury. 2,11

The incidence of vascular injury in knee dislocations ranges from less than $5 \%$ up to $65 \%$. A more recent systematic review of larger numbers of patients shows the overall incidence is 18 to $20 \% .^{18,19}$ Vascular injury is potentially limb threatening, so it is essential to evaluate the limb perfusion and identify the presence of vascular compromise. It can be done with several methods as follows: palpation of dorsalis pedis and posterior tibial pulses bilaterally and assessing for any asymmetry, performing arterial Doppler examination, assessing bilateral ankle-brachial-indices (ABI; the sensitivity of $A B I$ in detecting vascular injury requiring surgical intervention approaches is $100 \%$ if $A B I$ is $<0.9^{20}$ ), or performing angiography or CT angiography that currently are recommended for patients with insufficient distal perfusion or asymmetry in physical examination. ${ }^{21}$

The incidence of nerve injury associated with knee dislocation ranges from 4.5 to $40 \%$ and the peroneal nerve is the most commonly injured. ${ }^{2,21}$ Other potential complications associated with knee dislocations include compartment syndrome, deep vein thrombosis, and periarticular fractures.

Initial management of knee dislocation, after evaluation of neurovascular status, begins with an immediate closed reduction under anesthesia. After reduction, in absence of neurovascular injury, temporary stabilization of the knee can be achieved by nonoperative technique with the use of a hinge brace or, operative, with an external fixator which is indicated when there is an associated vascular repair, open injury, fasciotomy, or unstable reduction that cannot be maintained with the use of a hinged brace. It is mandatory to repeat physical examinations, including ABI measurement, approximately every 2 hours and continued for a minimum of 48 hours, to detect vascular lesions that develop in a delayed fashion. ${ }^{22}$ Subsequently, combined ligament injuries should be treated in consideration of patient age and activities of daily living.

The majority of knee dislocations can be initially treated with a closed reduction under anesthesia but occasionally, particularly with posterolateral knee dislocations, the reduction cannot be obtained due to capsule-ligament structures interposition inside the enlarged medial joint space. Clarke 23 in 1942 was the first to describe what is now known as the "dimple sign." The "dimple" or "pucker" sign is caused by invagination of the skin and soft tissues into the medial joint space and it is a sign that the medial femoral condyle has buttonholed through the medial joint capsule; it is known to be pathognomonic of irreducible knee dislocation and requires open reduction. Irreducible posterolateral knee dislocation is a rare but well-documented entity. ${ }^{3-8,12,14,24-29}$

Surgical repair or reconstruction of soft tissue and ligament at the time of open reduction can be performed, but 
treatment algorithm is variable in the case report. Malik et $\mathrm{al}^{30}$ have recently proposed an algorithm for the management of irreducible posterolateral knee dislocation without vascular injury.

All cases reported the treatment included arthroscopic or open reduction with repair of one or all torn ligament ${ }^{29}$ or staged multiligament reconstruction. In the two largest series reported the mean age was 37.8 and 46 years, ${ }^{29,31}$ and no patient was more than 61 years old, all with good bone quality.

The case we reported is the only one described in the literature of a very old patient (87 years) who sustained an irreducible posterolateral fracture dislocation of the knee and has undergone to open reduction and simultaneous knee replacement with rotating hinge prosthesis.

Although arthroplasty has become an option with proven beneficial for fracture in shoulder, elbow and hip joint in the elderly population, this has not been the case for knee arthroplasty in the presence of a periarticular fractures around the knee that, the geriatric population is associated with a high incidence of postoperative complications and poor results than arthroplasties performed in patients with primary osteoarthritis (OA). ${ }^{32}$ Knee arthroplasty in the setting of complex distal femur or proximal tibial fracture in the elderly is a technically demanding surgery, that require thorough knowledge of the basic rules of revision surgery regarding the choice of constraint, planning of joint-line restoration and component rotation, bone defect filling, and implant fixation. ${ }^{33}$

Potential indications for primary total knee replacement (TKR) after around the knee fractures are existing OA, an intra-articular fracture with severe soft-tissue injuries, osteoporosis with articular impression and bone defects, and patients with insufficient compliance to allow partial or nonweight-bearing treatment. ${ }^{34}$ Most of the cases published in literature about total joint replacement in fractures described old patients with comminute fractures and osteoporotic bone. This especially described in proximal humeral and femoral fractures. Instead few articles have been published in articular fracture of old people with severe capsuloligament injuries and consequent instability. Most of the papers have been written in elbow fractures, especially in case of terrible triad injury. The indication to elbow arthroplasty is suggested in old and osteoporotic patients. ${ }^{35}$

In general, but especially in our case, TKR allows elimination of fracture healing issues (failure of fixation and loss of reduction), knee stiffness due to arthrofibrosis, secondary $\mathrm{OA}$ and offers advantages in terms of early mobilization, immediate weight bearing, and faster rehabilitation combined with a decreased probability of reoperation. The goal is to limit functional impairment, loss of autonomy, and to allow these patients to return as fast as possible to their prefracture day living activities.

Although there is limited evidence in the literature, there is a role for acute knee arthroplasty in distal femoral or proximal tibial fractures in the elderly patients. This kind of treatment has shown satisfactory survival and revision rates, enabling immediate full weight bearing, rapid mobilization, and early discharge of patients. The risk of complications associated with primary TKR is higher than those reported after TKR due to primary OA but lower than those reported after TKR due to secondary OA. ${ }^{36,37}$

\section{Conclusion}

In conclusion, posterolateral dislocations of the knee are very rare injury that can be missed or misdiagnosed. The anteromedial "pucker" sign that is accentuated by knee flexion and the irreducibility by closed manipulation because of soft tissue incarceration are the main findings of this uncommon entity. Open reduction should be performed as soon as vascular compromise is excluded. Combined ligament injuries should be treated after the reduction, in consideration of patient age and activities of daily living.

\section{Ethical Approval}

The patient gave written informed consent prior being included in the case report.

\section{Funding \\ None.}

\section{Conflict of Interest}

None declared.

\section{References}

1 Frassica FJ, Sim FH, Staeheli JW, Pairolero PC. Dislocation of the knee. Clin Orthop Relat Res 1991;(263):200-205

2 Wascher DC, Dvirnak PC, DeCoster TA. Knee dislocation: initial assessment and implications for treatment. J Orthop Trauma 1997; 11(07):525-529

3 Huang FS, Simonian PT, Chansky HA. Irreducible posterolateral dislocation of the knee. Arthroscopy 2000;16(03):323-327

4 Silverberg DA, Acus R. Irreducible posterolateral knee dislocation associated with interposition of the vastus medialis. Am J Sports Med 2004;32(05):1313-1316

5 Bistolfi A, Massazza G, Rosso F, et al. Non-reducible knee dislocation with interposition of the vastus medialis muscle. J Orthop Traumatol 2011;12(02):115-118

6 Braun DT, Muffly MT, Altman GT. Irreducible posterolateral knee dislocation with entrapment of the adductor magnus tendon and medial skin dimpling. J Knee Surg 2009;22(04):366-369

7 Baxamusa TH, Galloway MT. Irreducible knee dislocations secondary to interposed menisci. Am J Orthop 2001;30(02):141-143

8 Pugh KJ, Wilber JH. Irreducible fracture dislocation of the knee. J Orthop Trauma 1996;10(05):359-362

9 Shetty GM, Wang JH, Kim SK, et al. Incarcerated patellar tendon in Hoffa fracture: an unusual cause of irreducible knee dislocation. Knee Surg Sports Traumatol Arthrosc 2008;16(04):378-381

10 Green RN, Pullagura MK, Holland JP. Irreducible fracture-dislocation of the knee. Acta Orthop Traumatol Turc 2014;48(03): 363-366

11 Schenck RJr. Classification of knee dislocations. Oper Tech Sports Med 2003;11(03):193-198

12 Tateda S, Takahashi A, Aizawa T, Umehara J. Closed reduction of "irreducible" posterolateral knee dislocation - a case report. J Orthop Case Rep 2016;6(02):20-23

$13 \mathrm{Xu} \mathrm{H}, \mathrm{Guo}$ R, Xu B. An irreducible posterolateral knee dislocation: diagnosis, arthroscopic view, and treatment. Am J Emerg Med 2016;34(11):2256.e3-2256.e4 
14 Urgüden M, Bilbaşar H, Ozenci AM, Akyildiz FF, Gür S. Irreducible posterolateral knee dislocation resulting from a low-energy trauma. Arthroscopy 2004;20(Suppl 2):50-53

15 Vaidya R, Roth M, Nanavati D, et al. Knee dislocation from minor trauma in morbidly obese patients. Orthop J Sports Med 2013. Doi: $10.1177 / 2325967113500081$

16 Marin EL, Bifulco SS, Fast A. Obesity. A risk factor for knee dislocation. Am J Phys Med Rehabil 1990;69(03):132-134

17 Fanelli GC, Orcutt DR, Edson CJ. The multiple-ligament injured knee: evaluation, treatment, and results. Arthroscopy 2005;21 (04):471-486

18 Keating JF. Acute knee ligament injuries and knee dislocation. In: Bentley G. European Surgical Orthopedics and Traumatology. The EFORT Textbook. Berlin, Germany: Springer; 2014:2949-2971

19 Medina O, Arom GA, Yeranosian MG, Petrigliano FA, McAllister DR. Vascular and nerve injury after knee dislocation: a systematic review. Clin Orthop Relat Res 2014;472(09):2621-2629

20 Mills WJ, Barei DP, McNair P. The value of the ankle-brachial index for diagnosing arterial injury after knee dislocation: a prospective study. J Trauma 2004;56(06):1261-1265

21 Lachman JR, Rehman S, Pipitone PS. Traumatic knee dislocations: evaluation, management, and surgical treatment. Orthop Clin North Am 2015;46(04):479-493

22 Barnes CJ, Pietrobon R, Higgins LD. Does the pulse examination in patients with traumatic knee dislocation predict a surgical arterial injury? A meta-analysis. J Trauma 2002;53(06):1109-1114

23 Clarke HO. Dislocation of the knee-joint with capsular interposition. Proc R Soc Med 1942;35:759

24 Saini R, Mootha AK, Goni VG, Dhillon MS. Neglected irreducible posterolateral knee dislocation. Indian J Orthop 2010;44(04): 468-470

25 Solarino G, Notarnicola A, Maccagnano G, Piazzolla A, Moretti B. Irreducible posterolateral dislocation of the knee: a case report. Joints 2015;3(02):91-96

26 Woon CY, Hutchinson MR. Posterolateral dislocation of the knee: recognizing an uncommon entity. World J Orthop 2016;7(06): 401-405
27 Dubberley J, Burnell C, Longstaffe A, MacDonald PB. Irreducible knee dislocation treated by arthroscopic debridement. Arthroscopy 2001;17(03):316-319

28 Al-Juhani W, Ahmed B, AlMugren T. Irreducible knee dislocation with vastus medialis muscle interposition. Knee Surg Relat Res 2019;31(01):72-75

29 Hongwu Z, Li J. One-stage arthroscopic reduction combined with multiligament reconstruction or repair for irreducible posterolateral knee dislocation: a retrospective case series with minimum 2-year follow-up. J Knee Surg 2018;31(10): 1015-1021

30 Malik SS, MacDonald PB. The irreducible knee dislocation. J Knee Surg 2020;33(04):328-334

$31 \mathrm{Gu} \mathrm{MQ}$, Deng L, Liu Y. Posterolateral dislocation of the knee joints: analysis of 9 cases. Chin J Traumatol 2004;7(04): 210-216

32 Scott CE, Davidson E, MacDonald DJ, White TO, Keating JF. Total knee arthroplasty following tibial plateau fracture: a matched cohort study. Bone Joint J 2015;97-B(04):532-538

33 Parratte S, Ollivier M, Argenson JN. Primary total knee arthroplasty for acute fracture around the knee. Orthop Traumatol Surg Res 2018;104(1S):S71-S80

34 Softness KA, Murray RS, Evans BG. Total knee arthroplasty and fractures of the tibial plateau. World J Orthop 2017;8(02): 107-114

35 Ramsey ML, Adams RA, Morrey BF. Instability of the elbow treated with semiconstrained total elbow arthroplasty. J Bone Joint Surg Am 1999;81(01):38-47

36 Senthilkumaran S, MacDonald DRW, Rankin I, Stevenson I. Total knee arthroplasty for distal femoral fractures in osteoporotic bone: a systematic literature review. Eur J Trauma Emerg Surg 2019;45(05):841-848

37 Tapper V, Toom A, Pesola M, Pamilo K, Paloneva J. Knee joint replacement as primary treatment for proximal tibial fractures: analysis of clinical results of twenty-two patients with mean follow-up of nineteen months. Int Orthop 2020;44(01): 85-93 